\title{
Validation of the clinical tuberculosis screening algorithm used in Nigerian national tuberculosis control programme for screening people living with HIV
}

Emmanuel N. Aguwa ${ }^{1 *}$, Chika N. Onwasigwe ${ }^{1}$, Joseph N. Chukwu ${ }^{2}$, Daniel C. Oshi ${ }^{2}$, Charles C. Nwafor ${ }^{2}$, Babatunde I. Omotowo ${ }^{2}$, Anne C. Ndu ${ }^{1}$, Anthony O. Meka ${ }^{2}$, Osa-Eloka C. Ekwueme ${ }^{1}$, Nwachukwu C. Ugwunna ${ }^{1}$, Moses C. Anyim ${ }^{2}$

${ }^{1}$ Department of Community Medicine, University of Nigeria, Nsukka, Nigeria; ${ }^{*}$ Corresponding Author: enaguwa@yahoo.com

${ }^{2}$ German Leprosy and Tuberculosis Relief Association, Enugu, Nigeria

Received 25 July 2013; revised 25 August 2013; accepted 15 September 2013

Copyright (C) 2013 Emmanuel N. Aguwa et al. This is an open access article distributed under the Creative Commons Attribution License, which permits unrestricted use, distribution, and reproduction in any medium, provided the original work is properly cited.

\section{ABSTRACT}

Background: In high HIV prevalence, tuberculosis diagnosis is challenging. Some countries hence use clinical algorithms to screen for tuberculosis in People Living with HIV (PLHIV). Objectives: The aim of the study was to validate the national algorithm for clinical tuberculosis screening of persons living with HIV who attend comprehensive HIV clinics. Methods: A crosssectional study of PLHIV who presented with cough of at least 2 weeks duration between 2009 and 2011 at St Patrick's Hospital, Ebonyi State, Nigeria. Sputum smear microscopy for acid fast bacilli was obtained from the participants. Results: Three hundred and twelve PLHIV were studied: 146 (46.8\%) males and $166(53.2 \%)$ females. Only $55(17.6 \%)$ of the participants had smear positive pulmonary tuberculosis. Weight loss $\left(\chi^{2}=2.33 ; \mathbf{P}=0.127\right)$, hemoptysis $\left(\chi^{2}=0.03\right.$; $\mathbf{P}=0.864)$, night sweats $\left(\chi^{2}=1.52 ; \mathbf{P}=0.218\right)$, fever $\left(\chi^{2}=3.49 ; \mathbf{P}=0.06\right)$, anorexia $\left(\chi^{2}=0.49 ; \mathbf{P}=\right.$ $0.484)$, chest pain $\left(\chi^{2}=2.48 ; \mathrm{P}=0.115\right)$, breathlessness $\left(\chi^{2}=0.63 ; P=0.426\right)$ were not significant in PLHWA with/without pulmonary tuberculosis. Cough, fever, night sweat and weight loss combined gave a sensitivity of $97.0 \%$, specificity of $10.9 \%$, negative predictive value (NPV) of $93.3 \%$ and positive predictive value (PPV) of $\mathbf{2 1 . 8 \%}$. Conclusion: Findings suggest that though national screening algorithm is a valid tool to screen for tuberculosis in PLHIV, it will lead to many false positive results.

Keywords: HIV; Tuberculosis; PLHIV; Screening Tool Validation

\section{INTRODUCTION}

Since the onset of Human Immunodeficiency Virus (HIV), the incidence of tuberculosis (TB) has been increasing. Globally about 9 million new cases of active TB occur annually and most of these are in sub-Saharan Africa [1]. Indeed thirteen of the 15 countries with the highest incidence rates of TB per capita lie within this region [2]. The special relationship between TB and HIV has long been established: HIV infected persons are twenty times more likely to come down with active TB [3] and TB is the leading cause of death among HIV-infected persons [4]. However, although over $60 \%$ of new cases of pulmonary TB in some developing countries are co-infected with HIV [2], only fewer than $50 \%$ of these cases get diagnosed before death [5].

In Nigeria, like in many developing countries, reports show that case detection rate of $45 \%$ in 2011 [6] is lower than WHO target for TB control. This is because diagnosis of TB in HIV infected persons is sometimes difficult $[7,8]$. Patients who are dually infected with HIV and TB are occasionally known to have atypical clinical and radiological presentations for TB. They are also more likely to have extra-pulmonary TB and smear negative pulmonary TB [9]. This delayed diagnosis of TB may contribute to the high mortality of TB in HIV infected persons [10]. Unfortunately, though increasing numbers of 
pulmonary TB patients are known to have false negative results on smear microscopy especially in the presence of the late stages of HIV, use of sputum smear microscopy remains the hallmark of diagnosis in resource-poor countries. Several studies have therefore tried to establish clinical algorithms for diagnosis of pulmonary TB [11$16]$.

Study objective: To validate the National Algorithm for Clinical TB Screening for People Living with HIV/ AIDS (PLHIV).

\section{MATERIALS AND METHODS}

The study area was St. Patrick's Hospital (Mile 4) Abakiliki, the capital of Ebonyi State. Ebonyi State is one of the five states in South East Nigeria and according to 2006 National Population Census, has a population of 2,173,501 [17]. It started managing TB patients in 1969 and currently has 311 beds for treating TB. It is the largest tuberculosis centre in southern Nigeria and is one of the first to pilot TB/HIV collaborative interventions in National Tuberculosis and Leprosy Control Programme (NTBLCP).

The study was a descriptive cross-sectional study carried out between $1^{\text {st }}$ quarter 2009 and $1^{\text {st }}$ quarter 2011 at St. Patrick's (Mile 4) Hospital. A calculated sample size of 312 PLHIV was used for the study. Institutional Review Board approval for the study was obtained from the Ethics Committee of University of Nigeria Teaching Hospital (UNTH) Enugu. All new PLHIV who reported for HIV treatment at St Patrick's hospital and who gave their informed consent were enrolled in the study. Written informed consent was obtained from each participant. Participants were interviewed using a pre-tested interviewer-administered, semi-structured questionnaire. Data on presenting symptoms and signs were collected. The patients were taught on how to produce and collect sputum which was subsequently sent to a quality assured microbiology laboratory for Ziehl Neelson sputum smear microscopy. The reagents used were methylene blue, acid alcohol and strong Carbol Fuchsin obtained from Svizera Europe GCC Diagnostics Gailand Chemical Company Sandycroft Deeside UK Lot No. 1010. Three sputum samples were collected: spot, early morning and spot.

On the first day, participant provided an "on the spot" sputum sample under supervision. Then participant was given sputum container to take home for an "early morning” sample the following morning. On the second day, participant produced and brought the "early morning sample" to the lab as the second sputum sample. Same day participant provided another "on the spot" sample under supervision. This became the third sputum sample. Where only one sputum sample out of the three samples was positive, three more sputum samples were collected.
Each patient's sputum containers were labelled "real time" using his initials, date of collection and date of birth. This was to avoid mix up in the samples and also to ensure confidentiality. The laboratory was subjected to internal and external quality checks.

Data analysis

Sputum AFB grading was analyzed based on the WHO recommended grading: [18]

No AFB............ AF AFB observed per 100 fields of the stained slide

Scanty...........1 to 9 AFB per 100 fields seen

$+\ldots \ldots \ldots \ldots \ldots . . .10$ - 99 AFB per 100 fields seen

$++\ldots \ldots \ldots \ldots \ldots . . . .1-10$ AFB per 50 fields seen

$+++\ldots \ldots \ldots \ldots \ldots . . . .10$ AFB per field in 20 fields seen

Interpretation of the results was based on the National guideline.

Patients were classified as smear-positive when two sputum specimens are positive for acid-fast bacilli (AFB). Where only one sputum specimen was positive, three more sputum examinations were carried out. Where one other was positive, the patient was classified as smearpositive TB. Data entry and Analysis: Data were entered and analyzed using Statistical Package for Social Sciences (SPSS) version 16. Discrete variables were tested for significance with Chi square. Regression of the variables were then done to predict the outcome i.e. likelihood of clinical symptoms suggestive of pulmonary TB.

\section{RESULTS}

A total of 312 PLHIV were studied: 146 (46.8\%) were males while 166 (53.2\%) were females. Most of the participants were married and had at least primary level of education. Most of the patients are within the age group of 21 - 40 years. Only 55 (17.6\%) of the participants had smear positive pulmonary TB. There was no significant difference in the sex $(\mathrm{P}=0.117)$ and age groups $(\mathrm{P}=$ 0.428) in PLHIV with/without TB (Table 1). In Table 2,

Table 1. Sex and age range of PLHIV with and without pulmonary tuberculosis.

\begin{tabular}{lcc}
\hline \multirow{2}{*}{ Variables } & \multicolumn{2}{c}{ Tuberculosis Diagnosis } \\
\cline { 2 - 3 } & Positive (\%) & Negative (\%) \\
\hline - Male & $31(56.4)$ & $115(44.7)$ \\
- Female & $24(43.6)$ & $142(55.3)$ \\
$\quad$ Total & $55(100.0)$ & $257(100.0)$ \\
& $\chi^{2}=2.46, \mathrm{P}=0.117$ & \\
Age range (years) & & \\
- 20 or less & $2(3.6)$ & $19(7.4)$ \\
- 21 - 40 & $40(72.7)$ & $165(64.2)$ \\
- 41 - 60 & $11(20.1)$ & $68(26.5)$ \\
- 61 and above & $2(3.6)$ & $5(1.9)$ \\
& $55(100.0)$ & $257(100.0)$ \\
& $\chi^{2}=2.77, \mathrm{P}=0.428$ & \\
\hline
\end{tabular}


previous history of TB or family history of TB did not significantly contribute to presence of TB in PLHIV (P = 0.292 and $\mathrm{P}=0.493$ respectively).

Similarly, all presenting symptoms in PLHIV with and without TB were similar $(\mathrm{P}>0.05)$ Table 3 . None of the symptoms individually was significantly more in PLHIV with TB and those without TB i.e. presence of each symptom alone did not indicate presence of $\mathrm{TB}$ in PLHIV. However, if symptoms like cough, fever, night sweats and weight loss are combined to make a diagnosis of TB in PLHIV, there was low specificity (10.9\%) and high sensitivity (97.0\%). It was also of high negative predictive value (NPV) (93.3\%) but a low positive predictive value (PPV) (21.8\%) Table 4.

\section{DISCUSSION}

Among the 312 persons living with HIV/AIDS studied, less than a quarter (17.6\%) were found to have smear

Table 2. History of tuberculosis in PLHIV with or without current tuberculosis.

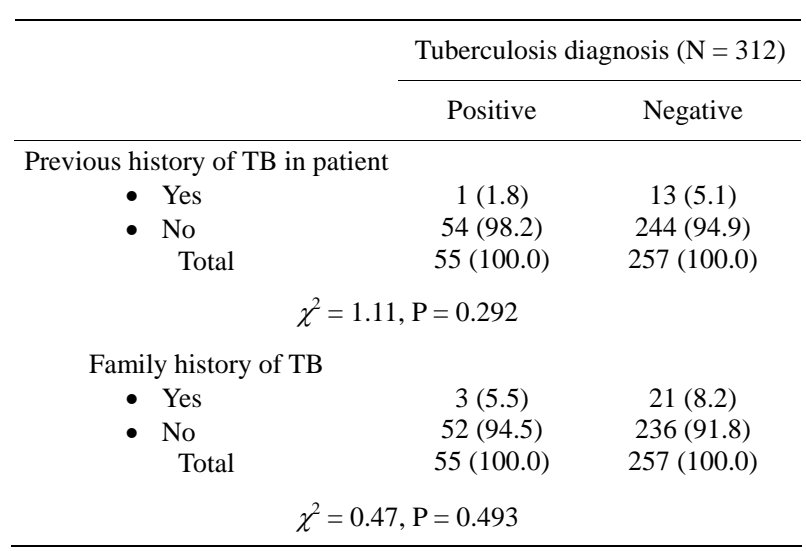

Table 3. Presenting symptoms in PLHIV with or without tuberculosis.

\begin{tabular}{|c|c|c|c|}
\hline \multirow{2}{*}{$\begin{array}{l}\text { Presenting } \\
\text { Symptoms }\end{array}$} & \multicolumn{2}{|c|}{ Tuberculosis diagnosis $(\mathrm{N}=312)$} & \multirow[b]{2}{*}{$\chi^{2}$ (P value) } \\
\hline & $\begin{array}{c}\text { Positive } n 1=55 \\
\text { (\%) }\end{array}$ & $\begin{array}{c}\text { Negative n2 }=257 \\
\text { (\%) }\end{array}$ & \\
\hline Cough & $55(100.0)$ & $257(100.0)$ & \\
\hline Weight loss & $53(96.4)$ & 231 (89.9) & $2.33(0.127)$ \\
\hline $\begin{array}{l}\text { Coughing out } \\
\text { blood }\end{array}$ & $13(23.6)$ & $58(22.6)$ & $0.33(0.864)$ \\
\hline Night sweat & $36(65.5)$ & 145 (56.4) & $1.52(0.218)$ \\
\hline Night fever & $47(85.5)$ & 189 (73.5) & $3.49(0.06)$ \\
\hline Loss of appetite & $33(60.0)$ & $167(65.0)$ & $0.49(0.484)$ \\
\hline $\begin{array}{l}\text { Excessive } \\
\text { tiredness }\end{array}$ & 30 (54.5) & 141 (54.9) & $0.00(0.966)$ \\
\hline Chest pain & $46(83.6)$ & 189 (73.5) & $2.48(0.115)$ \\
\hline Breathlessness & $30(54.5)$ & 125 (48.6) & $0.63(0.426)$ \\
\hline
\end{tabular}

Table 4. Predictive value of the clinical algorithm of PLHIV for presence of pulmonary tuberculosis.

\begin{tabular}{|c|c|c|c|c|}
\hline $\begin{array}{c}\text { Symptoms } \\
\text { Present }\end{array}$ & Specificity & Sensitivity & $\begin{array}{c}\text { Negative } \\
\text { Predictive } \\
\text { Value (NPV) }\end{array}$ & $\begin{array}{c}\text { Positive } \\
\text { Predictive } \\
\text { Value (PPV) }\end{array}$ \\
\hline $\begin{array}{c}\text { Cough }+ \text { fever }+ \\
\text { night sweats }+ \\
\text { weight loss }\end{array}$ & $10.9 \%$ & $97.0 \%$ & $93.3 \%$ & $21.8 \%$ \\
\hline
\end{tabular}

positive tuberculosis. Similar prevalence of TB was obtained among PLHIV in some other Nigerian studies [19, 20]. The commonest symptom present in all the PLHIV with or without pulmonary tuberculosis is cough without blood. Other associated symptoms such as weight loss, night sweats, fever, loss of appetite, excessive tiredness, chest pain and breathlessness did not vary significantly $(\mathrm{P}>0.05)$ between the PLHIV with or without TB coinfection.

The Word Health Organization recommends tuberculosis screening at the time that HIV infection is diagnosed, before the initiation of anti-retroviral therapy and isoniazid preventive therapy and regularly thereafter [21]. The presence of combination of cough, fever, night sweats and weight loss was found to be specific for tuberculosis in $10.9 \%$ and sensitive in $97.0 \%$ of the cases. The high sensitivity of this clinical algorithm as found in this study means there is high true positive and low false negative results. This is particularly important for making further evaluation to confirm the presence or absence of tuberculosis since a missed opportunity in diagnosis of tuberculosis in a person with HIV infection can have serious implications and consequences in both the control efforts and in the management of dually infected individuals i.e. failure to suspect or diagnose pulmonary TB or inadequately isolate the organism will result in disease transmission. This fact was earlier observed by Tattevin et al. [13] and that led to their advocating for improved interpretation of clinical and radiological data of patients on admission to improve adequacy of respiratory isolation and to reduce missed opportunity in diagnosis of tuberculosis among dually infected individuals. Similarly, World Health Organization and Center for Disease Control developed an algorithm for diagnosis of TB in PLHIV which included the presence of the following symptoms: cough (regardless of duration), fever, night sweats and weight loss. Their findings, like that of present study had high sensitivity (79\%) but low specificity (56\%) [22].

The low specificity observed in this study indicates that there is low true negative and high false positive result i.e. many cases may be recorded as being dually infected when actually there was no TB. However, this is overridden by the high sensitivity since the group of persons not ruled out by this algorithm will be subjected 
to sputum microscopic examination for confirmation. A study of 1748 persons with HIV infection from eight outpatient clinics in Cambodia, Thailand and Vietnam to determine the algorithm for tuberculosis screening and diagnosis in people with HIV was conducted by Kevin et al. [19] The result similarly showed a high sensitivity of $93 \%$ and low specificity of $36 \%$ for tuberculosis for the presence of cough, fever and night sweats. Presence of cough alone had a sensitivity of $22 \%$ to $33 \%$ for detecting tuberculosis [21]. In the study by Tattevin et al., they proposed a predictive model based on the combination of medical history, social factors, symptoms and Chest Xray pattern [13].

In present study, no significant difference $(\mathrm{P}>0.05)$ was noted in the presence or absence of previous personal and family history of tuberculosis among the PLHIV with or without tuberculosis. The high sensitivity (97\%) and negative predictive value of $93.3 \%$ observed in this study are similar to many other studies where two or more symptoms were used to formulate a predictive model for active tuberculosis in PLHIV. A study conducted in Uganda to predict the presence of active tuberculosis in a population of HIV-infected adults receiving care before accessing ART used the presence of cough of greater than 3 weeks duration, fever of greater than 4 weeks duration, lymphadenopathy, or reduced body mass index (BMI). The result showed 99\% sensitivity and $66 \%$ specificity with a NPV of $100 \%$ for predicting active TB at the initial assessment for ART. For patients receiving ART for more than 3 months the presence of either a cough of greater than 3 weeks or general weakness of greater than 2 weeks was $100 \%$ sensitive and $66 \%$ specific with a negative predictive value (NPV) of 100\% for active TB diagnosis. The high NPV implies that the algorithm could be used to efficiently identify PLHIV who are unlikely to have active TB [14]. Furthermore, a study in South Africa observed that among patients with advanced HIV disease not taking anti-retroviral therapy, a screening instrument of two or more symptoms was $100 \%$ sensitive and $88 \%$ specific with an NPV of $100 \%$ [15]. Similar studies in Tanzania and Burundi showed that the combination of any two of four symptoms (cough greater than 3 weeks duration, chest pain greater than 15 days, absence of expectoration or absence of shortness of breath) among adults had a sensitivity of $85 \%$ and an NPV of $90 \%$ [16].

The findings in this study can be used to divide the population of persons living with HIV/AIDS clinically into two groups: those who do not have tuberculosis and those who need further evaluation to confirm the presence or absence of tuberculosis in line with the goal of tuberculosis screening. The high sensitivity result obtained in this study is in line with the requirement that a good screening test be sensitive enough to avoid missed diagnosis of tuberculosis in PLHIV with its attendant grave consequences in terms of disease transmission and increased morbidity and mortality. The high negative predictive value of the symptoms as also found in this study also assures that those screened out do not actually have the tuberculosis disease.

\section{CONCLUSION AND RECOMMENDATION}

This study suggests that the algorithm of using presence of cough, fever, night sweats and weight loss to screen for TB in PLHIV will pick up most PLHIV with active pulmonary TB. It will, however, also pick up a lot of false positive cases due to its low specificity. Hence, it is recommended that as presently directed, all PLHIV should be screened for TB using the screening algorithm.

\section{REFERENCES}

[1] World Health Organization (2011) Global tuberculosis control. WHO Report 2011.

[2] Corbett, E.L., Watt, C.J., Walker, N., et al. (2003) The growing burden of tuberculosis. Global trends and interactions with the HIV epidemic. Archives of Internal Medicine, 163, 1009-1021. http://dx.doi.org/10.1001/archinte.163.9.1009

[3] Getahun, H., Gunneberg, C., Granich, R. and Nunn, P. (2010) HIV infection-associated tuberculosis: The epidemiology and the response. Clinical Infectious Diseases, 50, S201-S207. http://dx.doi.org/10.1086/651492

[4] Harries, A.D., Hargreaves, N.J., Kemp, J., et al. (2001) Deaths from tuberculosis in sub-Saharan African countries with a high prevalence of HIV-1. Lancet, 357, 15191523. http://dx.doi.org/10.1016/S0140-6736(00)04639-0

[5] Rana, F.S., Hawken, M.P., Mwachari, C., et al. (2000) Autopsy study of HIV-1-positive and HIV-1-negative adult medical patients in Nairobi, Kenya. Journal of Acquired Immune Deficiency Syndromes, 24, 23-29.

[6] World Bank (2012) Tuberculosis case detection rate (\%, all forms). The World Bank Data. http://data.worldbank.org/indicator/SH.TBS.DTEC.ZS

[7] Sharma, S.K., Mohan, A. and Kadhiravan, T. (2005) HIVTB co-infection: Epidemiology, diagnosis \& management. Indian Journal of Medical Research, 121, 550-567.

[8] Ha, D.T.M., Lan, N.T.N., Kiet, V.S., Wolbers, M., Hang, H.T.T., Day, J., Hien, N.Q., Tien, N.A., et al. (2010) Diagnosis of pulmonary tuberculosis in HIV-positive patients by microscopic observation drug susceptibility assay. Journal of Clinical Microbiology, 48, 4573-4579. http://dx.doi.org/10.1128/JCM.00687-10

[9] Sterling, T.R., Pham, P.A. and Chaisson, R.E. (2010) HIV infection-related tuberculosis: Clinical manifestations and treatment. Clinical Infectious Diseases, 50, S223-S230. http://dx.doi.org/10.1086/651495

[10] Cain, K.P., Anekthanaon, T., Burapat, C., et al. (2009) Causes of death in HIV-infected persons who have tu- 
berculosis, Thailand. Emerging Infectious Diseases, 15, 258-264. http://dx.doi.org/10.3201/eid1502.080942

[11] Conde, M.B., Figueira, C.M., Moraes, R., Fonseca, L.S., DeRiemer, K. and Kritski, L.(1999) Predictive value of acid fast smear for detection of mycobacterium tuberculosis in respiratory specimen in reference center of HIV/ AIDS in Rio de Jeneriro. Memorials do instituto Oswaldo Cruz On-Line, 94, 787-790.

[12] Weber, A.M., Areerat, P., Fischer, J.E., Thamthitiwat, S., Olsen, S.J. and Varma, J.K. (2008) Factors associated with diagnostic evaluation for tuberculosis adults hospitalized with clinical pneumonia in Thailand. Infection Control and Hospital Epidemiology, 29, 648-657. http://dx.doi.org/10.1086/588684

[13] Tattevin, P., Casalino, E., Fleury, L., Egmann, G., Ruel, M. and Bouvet, E. (1999) The validity of medical history, clinical symptoms and chest radiography in predicting pulmonary tuberculosis. Chest, 115, 1248-1253. http://dx.doi.org/10.1378/chest.115.5.1248

[14] Were, W., Moore, D., Ekwaru, P., Mwima, G., Bunnell, R., Kaharuza, F., Rutherford, G. and Mermin, J. (2009) A simple screening tool for active tuberculosis in HIV-infected adults receiving antiretroviral treatment in Uganda. The International Journal of Tuberculosis and Lung Disease, 13, 47-53.

[15] Mohammed, A., Ehrlich, R., Wood, R., Cilliers, F. and Maartens G. (2003) Screening for tuberculosis in adults with advanced HIV disease prior to preventive therapy. The International Journal of Tuberculosis and Lung Disease, 8, 792-795.

[16] Samb, B., Henzel, D., Daley, C.L., Mugusi, F., Niyongabo, T., Mlika-Cabanne, N., Aubry, P., Mbaga, I., Larouze, B. and Murray, J.F. (1997) Methods for diagnosing tuberculosis among in-patients in eastern Africa whose sputum smears are negative. The International Journal of Tuberculosis and Lung Disease, 1, 25-30.

[17] Federal Republic of Nigeria (2006) Population census. National Bureau of Statistics. www.nigeriastat.gov.ng

[18] Weyer, K., De Kantor, I.N., Kim, S.J. and Frieden, T. (1998) Laboratory services in tuberculosis control. WHO, Geneva, 43.

[19] Olaniran, O., Hassan-Olajokun, R.E., Oyovwevotu, M.A. and Agunlejika, R.A. (2011) Prevalence of tuberculosis among HIV/AIDS patients in Obafemi Awolowo University Teaching Hospital Complex, ILE-IFE Nigeria. International Journal of Biological \& Medical Research, 2, 874-877.

[20] Iliyasu, Z. and Babashani, M. (2009) Prevalence and predictors of tuberculosis co-infection among HIV-seropositive patients attending the Aminu Kano Teaching Hospital, Northern Nigeria. Journal of Epidemiology, 19, 81-87. http://dx.doi.org/10.2188/jea.JE20080026

[21] Kevin, P.C., Kimberly, D.M., Charles, M.H., Patama, M., Theerawit, T. and Nong, K. (2010) An algorithm for TB screening and diagnosis in people with HIV. The New England Journal of Medicine, 362, 31-40.

[22] Getahun, H., Kittikraisak, W., Heilig, C.M., Corbett, E.L., Ayles, H., Cain, K.P., et al. (2011) Development of a standardized screening rule for tuberculosis in people living with HIV in resource-constrained settings: Individual participant data meta-analysis of observational studies. PLoS Medicine, 8, e1000391. http://dx.doi.org/10.1371/journal.pmed.1000391 